\title{
2012 Cole Prize in Algebra
}

ALEXANDER S. MERKURJEV received the 2012 AMS Frank Nelson Cole Prize in Algebra at the 118th Annual Meeting of the AMS in Boston in January 2012.

\section{Citation}

The 2012 Frank Nelson Cole Prize in Algebra is awarded to Alexander S. Merkurjev of the University of California, Los Angeles, for his work on the essential dimension of groups.

The essential dimension of a finite or of an algebraic group $G$ is the smallest number of parameters needed to describe $G$-actions. For instance, if $G$ is the symmetric group on $n$ letters, this invariant counts the number of parameters needed to specify a field extension of degree $n$, which is the algebraic form of Hilbert's thirteenth problem.

Merkurjev's papers ("Canonical $p$-dimension of algebraic groups”, with N. Karpenko, $A d v$. Math. 205 (2006), no. 2, 410-433; and "Essential dimension of finite $p$-groups", with N. Karpenko, Invent. Math. 172 (2008), no. 3, 491-508) introduce breakthrough new techniques to compute the essential dimension of $p$-groups. In his paper "Essential $p$-dimension of PGL $\left(p^{2}\right)$ " (Jour. Amer. Math. Soc. 23 (2010), no. 3, 693-712), which is a tour de force, Merkurjev calculates the essential dimension, localized at a prime $p$, of the group PGL $\left(p^{2}\right)$, which is bound up with understanding the structure of division algebras of dimension $p^{4}$ over general fields.

Merkurjev's unique style combines strength, depth, clarity, and elegance, and his ideas have had broad influence on algebraists over the last three decades.

\section{Biographical Sketch}

Alexander Merkurjev was born on September 25, 1955, in St. Petersburg (Leningrad), Russia. In 1977 he graduated from St. Petersburg University, and he received his Ph.D. there in 1979 under the direction of Anatoly Yakovlev. In 1983 he earned the Doctor of Sciences degree from St. Petersburg University for the work "Norm residue homomorphism of degree two". In 1983 Merkurjev won the Young Mathematician Prize of the St. Petersburg Mathematical Society for his work on algebraic $K$-theory. In 1995 he was awarded the Humboldt Prize. In 1977 Merkurjev became a professor at St. Petersburg University. Since 1997 he has been a professor at UCLA.

Merkurjev's interests lie in algebraic $K$-theory, algebraic groups, algebraic theory of quadratic forms, and essential dimension. He was an invited speaker at the International Congress of Mathematicians (Berkeley, 1986). Twice he has delivered an invited address at the European Congress of Mathematics $(1992,1996)$, and he was a plenary speaker in 1996 (Budapest).

\section{Response from Alexander S. Merkurjev}

It is a great honor and great pleasure for me to receive the 2012 Frank Nelson Cole Prize in Algebra. I would like to thank the American Mathematical Society and the Selection Committee for awarding the prize to me.

I am very grateful to my teacher, Andrei Suslin (he was awarded the Frank Nelson Cole Prize in Algebra in 2000). I also want to thank my parents, family, friends, and colleagues for their help and support over the years.

\section{About the Prize}

The Cole Prize in Algebra is awarded every three years for a notable paper in algebra published during the previous six years. The awarding of this prize alternates with the awarding of the Cole Prize in Number Theory, also given every three years. These prizes were established in 1928 to honor Frank Nelson Cole on the occasion of his retirement as secretary of the AMS after twentyfive years of service. He also served as editor-inchief of the Bulletin for twenty-one years. The Cole Prize carries a cash award of US\$5,000.

The Cole Prize in Algebra is awarded by the AMS Council acting on the recommendation of a selection committee. For the 2012 prize, the members of the selection committee were Robert L. Griess, János Kollár, and Parimala Raman.

Previous recipients of the Cole Prize in Algebra are: L. E. Dickson (1928), A. Adrian Albert (1939), Oscar Zariski (1944), Richard Brauer (1949), HarishChandra (1954), Serge Lang (1960), Maxwell A. Rosenlicht (1960), Walter Feit and John G. Thompson (1965), John R. Stallings (1970), Richard G. Swan (1970), Hyman Bass (1975), Daniel G. Quillen (1975), Michael Aschbacher (1980), Melvin Hochster (1980), George Lusztig (1985), Shigefumi Mori (1990), Michel Raynaud and David Harbater (1995), Andrei Suslin (2000), Aise Johan de Jong (2000), Hiraku Nakajima (2003), János Kollár (2006), and Christopher Hacon and James McKernan (2009). 